\title{
Local Treatment in Addition to Endocrine Therapy in Hormone Receptor-Positive and HER2- Negative Oligometastatic Breast Cancer Patients: A Retrospective Multicenter Analysis
}

\author{
Chihwan Cha ${ }^{\mathrm{a}}$ Sung Gwe Ahn ${ }^{\mathrm{a}}$ Tae-Kyung Yoo ${ }^{\mathrm{b}}$ Kun Min Kim ${ }^{\mathrm{c}}$ Soong June Bae ${ }^{\mathrm{a}}$ \\ Changik Yoon $^{\mathrm{a}}$ Soeun Park ${ }^{\mathrm{a}}$ Joohyuk Sohn ${ }^{\mathrm{c}}$ Joon Jeong ${ }^{\mathrm{a}}$ \\ aDepartment of Surgery, Gangnam Severance Hospital, Yonsei University College of Medicine, Seoul, South \\ Korea; ${ }^{b}$ Department of Surgery, Seoul St. Mary's Hospital, College of Medicine, The Catholic University of Korea, \\ Seoul, South Korea; ${ }^{\circ}$ Division of Medical Oncology, Department of Internal Medicine, Severance Hospital, Yonsei \\ University College of Medicine, Seoul, South Korea
}

\section{Keywords}

Oligometastasis · Hormone receptor-positive breast cancer · Local treatment

\begin{abstract}
Background: Recent trials have provided robust evidence demonstrating that endocrine therapy with/without targeted therapy, such as cyclin-dependent kinase $4 / 6$ inhibitors or mTOR (mammalian target of rapamycin) inhibitors, effectively halts disease progression in hormone receptor (HR)positive and human epidermal growth factor receptor 2 (HER2)-negative metastatic breast cancer. We investigated the survival impact of local treatment of metastases as a firstline treatment after metastasis in HR-positive and HER2-negative breast cancer patients with a very low metastatic volume. Materials and Methods: From a retrospectively constructed database for three institutes, we identified HRpositive and HER2-negative breast cancer patients with recurrent distant oligometastatic disease after initially curative treatment. De novo stage 4 patients were excluded, and only those with recurrent metastatic disease were included. Oligometastatic disease was defined as follows: (1) $\leq 2$ metastatic lesions in a single organ, (2) a maximal diameter $\leq 3 \mathrm{~cm}$, and (3) organ involvement, including the lung, liver, adrenal gland, bone, or distant lymph nodes. Local treatment com-
\end{abstract}

prised surgery or radiotherapy. Progression-free survival (PFS) and overall survival (OS) were investigated. Results: Forty-nine patients were included; 33 underwent local treatment. Of these 33 patients, 5 underwent surgical resection and 27 received radiotherapy. One patient underwent both surgical resection and radiotherapy. Median PFS was significantly longer among the patients with local treatment than among the patients without local treatment (30.0 vs. 18.0 months, $p=0.049$ ). In multivariate analysis, local treatment was shown to prolong PFS. However, median OS after metastasis did not differ with regard to local treatment (72.3 vs. 91.0 months, $p=0.272$ ). Conclusion: We showed that local treatment could positively affect disease progression in HRpositive and HER2-negative oligometastatic breast cancer.

(c) 2019 S. Karger AG, Basel

\section{Introduction}

While metastatic breast cancer is heterogeneous in nature, with presentations ranging from single lesions to involvement of multiple organs, survival outcomes of metastatic breast cancer patients have steadily improved [1,

C. Cha and S.G. Ahn contributed equally to this work. 
2]. Along with new targeted therapies that have been incorporated into daily practice [3-5], advances in diagnostic techniques that have made it possible to detect metastatic lesions early in the disease have contributed to increased survival with metastatic breast cancer [6].

In patients with metastatic breast cancer, treatment is primarily aimed at prolonging the duration of disease control without progression while minimizing side effects, and applications of surgery for metastatic lesions are limited. Recently, it has been hypothesized that early metastatic breast cancer could respond to treatment, and greater focus has been placed on curing the disease [7]. Explaining why early metastatic cancer could be treated effectively, the concept of oligometastasis was first presented by Hellman and Weichselbaum [8]. While definitions of oligometastasis currently differ between studies, it is generally considered to include fewer than 5 lesions, regardless of whether metastasis is limited to a single organ or occurs in multiple organs [9]. The incidence of oligometastasis was reported as $1-10 \%$ among patients with metastatic breast cancer [10]. Although retrospective studies are inevitably limited by selection bias, several studies have suggested that surgical removal of distant metastatic lesions might offer clinical benefits to those with oligometastases [1115]. In addition to surgical treatment, radiofrequency ablation (RFA) and radiotherapy might be effective in controlling metastatic lesions. Studies have shown that RFA is as effective as surgical resection for cases with metastatic tumors $<3 \mathrm{~cm}$ in size [16-18]. Also, it is reported that stereotactic body radiotherapy allows for the delivery of ablative doses of radiation for oligometastases, and is safe and effective [7], with local control rates of about $80 \%$ in oligometastatic cancer, including breast cancer [19-22].

In oligometastatic breast cancer patients receiving local treatment for their metastatic lesions, systemic therapy ought to follow metastasis-directed therapy or be administered simultaneously in order to control minimal residual disease. In patients with hormone receptor (HR)positive and human epidermal growth factor receptor 2 (HER2)-negative metastatic breast cancer, endocrine therapy with or without targeted therapy has been the standard of care as a first-line systemic therapy, with excellent results for disease palliation [23, 24]. Hence, if patients with HR-positive/HER2-negative oligometastatic disease are able to receive proper local control of their metastatic lesions, any residual systemic disease after local control might be more manageable.

In this study, we evaluated the survival impact of local treatment for distant metastatic lesions as a first-line treatment after metastasis in patients with recurrent HRpositive/HER2-negative metastatic breast cancer who had a very low metastatic volume.

\section{Subjects and Methods}

\section{Study Design}

From a retrospectively constructed database for three institutes (Gangnam Severance Hospital, Severance Hospital, and Seoul St. Mary's Hospital), we identified HR-positive/HER2-negative patients with oligometastatic breast cancer. All patients underwent curative surgery for their primary breast cancer and had recurrent disease identified as oligometastases. Patients with de novo metastases were excluded. The interval between surgery and metastasis was at least 1 year. Clinical data, including demographic features, pathologic characteristics, and survival outcomes, were collected retrospectively from the medical records of patients who had undergone surgery between 1993 and 2013.

\section{Definitions of Oligometastases}

We defined oligometastatic disease according to eligibility for curative resection or adequate local control. Oligometastatic disease was defined according to the following stringent criteria: (1) $\leq 2$ metastatic lesions in a single organ; (2) a maximal lesion diameter $\leq 3 \mathrm{~cm}$; and (3) organ involvement, including the lungs, liver, bone, or distant lymph nodes (neck node, mediastinal lymph node, and contralateral regional lymph node). Bone metastases were identified with at least two imaging modalities, including a bone scan, ${ }^{18}$ F-FDG-PET (18-fluorodeoxyglucose positron emission tomography), and MRI (magnetic resonance imaging). Other metastases were also diagnosed by radiologic modalities, including computed tomography scanning, MRI, and ${ }^{18} \mathrm{~F}-\mathrm{FDG}$-PET, with pathologic confirmation by needle biopsy or surgical resection, if possible.

\section{Definitions of Local Treatment}

Local treatment included surgical removal, radiotherapy, and RFA. Surgical removal was performed regardless of free margins. Radiotherapy included palliative and stereotactic body radiotherapy. The patients were divided into two groups: a "local treatment" group (metastasis-directed therapy plus endocrine treatments) and a "no local treatment" group (antiestrogen therapy alone without metastasis-directed therapy). The analyses were performed in two groups, regardless of the metastatic site.

\section{Patient Characteristics and Survival Outcomes}

Patients with oligometastases confined to one organ were included. The demographic data for the patients, including the age at diagnosis, follow-up periods, metastatic site, and size and number of metastases, were analyzed. Pathologic data, including the histologic type, initial pathologic stage of the tumor, number of pathologically positive axillary nodes, and Ki-67 labelling index, were collected. Treatment-related information, including the type of local treatment, type of first-line hormone therapy, duration of hormone therapy, and toxicity of metastasis-directed therapy, was analyzed.

Survival outcome data, including the time to disease progression after distant metastasis and the time to death after distant metastasis, were collected. The metastasis-free interval was investigated as well. The primary and secondary outcomes of the study were progression-free survival (PFS) and overall survival (OS), respectively. PFS was defined as the duration from diagnosis of the first distant metastasis to the time of disease progression. OS was defined as the duration from diagnosis of the first distant metastasis to the time of the last follow-up or death.

\section{Statistical Analyses}

Demographic characteristics are expressed as medians and percentages. A two-tailed Student $t$ test and the Mann-Whitney U test were used for comparisons between the two groups according to the 
Table 1. Patient characteristics at initial diagnosis

\begin{tabular}{|c|c|c|c|}
\hline & $\begin{array}{l}\text { Local } \\
\text { treatment } \\
(n=33)\end{array}$ & $\begin{array}{l}\text { No local } \\
\text { treatment } \\
(n=16)\end{array}$ & $\begin{array}{l}p \\
\text { value }\end{array}$ \\
\hline Age, years & 49.9 & 56.0 & 0.099 \\
\hline Follow-up, months & 101.6 & 105.6 & 0.791 \\
\hline Histologic type, $n(\%)$ & & & 0.219 \\
\hline Invasive ductal & $29(87.9)$ & $14(87.5)$ & \\
\hline Invasive lobular & $1(3.0)$ & $2(12.5)$ & \\
\hline Mucinous & $1(3.0)$ & & \\
\hline Other & $2(6.1)$ & & \\
\hline Initial stage, $n(\%)$ & & & 0.211 \\
\hline I & $9(27.3)$ & $2(12.5)$ & \\
\hline II & $12(36.4)$ & $10(62.5)$ & \\
\hline III & $12(36.4)$ & $4(25.0)$ & \\
\hline \multicolumn{4}{|l|}{ Number of pathologically } \\
\hline positive axillary nodes & 4.2 & 3.1 & 0.554 \\
\hline High Ki-67 index ${ }^{\mathrm{a}}, n(\%)$ & $5(15.2)$ & $2(12.5)$ & 0.850 \\
\hline \multicolumn{4}{|l|}{ Type of primary adjuvant } \\
\hline Tamoxifen & $14(42.4)$ & $4(25.0)$ & \\
\hline Letrozole & $5(15.2)$ & $4(25.0)$ & \\
\hline Anastrozole & $5(15.2)$ & $5(31.3)$ & \\
\hline Toremifene & $2(6.1)$ & $1(6.3)$ & \\
\hline Unknown & $6(18.2)$ & $2(12.5)$ & \\
\hline Not done & $1(3.0)$ & 0 & \\
\hline
\end{tabular}

${ }^{a}$ Ki-67 labelling index $\geq 20 \%$.
Table 2. Clinical and pathologic characteristics after first diagnosis of metastasis

\begin{tabular}{lccc}
\hline & $\begin{array}{l}\text { Local } \\
\text { treatment } \\
(n=33)\end{array}$ & $\begin{array}{l}\text { No local } \\
\text { treatment } \\
(n=16)\end{array}$ & $\begin{array}{l}p \\
\text { value }\end{array}$ \\
& & & \\
\hline $\begin{array}{l}\text { Type of first-line endocrine } \\
\text { treatment, } n(\%)\end{array}$ & $6(18.2)$ & 0 & 0.098 \\
Tamoxifen & $12(36.4)$ & $8(50.0)$ & \\
Letrozole & $5(15.2)$ & 0 & \\
Anastrozole & $8(24.2)$ & $5(31.3)$ & \\
Exemestane & $2(6.1)$ & $2(12.5)$ & \\
Fulvestrant & 0 & $1(6.3)$ & \\
Toremifene & & & $0.026^{*}$ \\
Metastatic site, $n$ (\%) & $23(69.7)$ & $9(56.3)$ & \\
Bone & $2(6.1)$ & $1(6.3)$ & \\
Lung & 0 & $4(25.0)$ & \\
Liver & $8(24.2)$ & $2(12.5)$ & \\
Distant lymph node & & 1.5 & 0.121 \\
Number of metastases & 1.0 & 1.26 & $0.005^{*}$ \\
Size of metastasis, cm & 1.98 & & \\
Type of local treatment, $n(\%)$ & & & \\
Radiation & $27(81.8)$ & & \\
Surgery & $5(15.2)$ & & \\
Both & $1(3.0)$ & & \\
Death & $6(18.2)$ & $1(6.3)$ & 0.263 \\
\hline
\end{tabular}

* $p<0.05$. " "Distant lymph node" includes the neck node, mediastinal lymph node, and contralateral regional lymph node.

\section{Treatments after Distant Metastasis}

Of the 33 patients in the local treatment group, 27 (81.8\%), 5 (15.2\%), and 1 underwent radiotherapy, surgery, and both radiation and surgery, respectively (Table 2). Twenty-two of the 23 patients with bone metastases underwent radiotherapy, and the remaining patient underwent surgery. Both of the 2 patients with lung metastases underwent surgery (wedge resection). Five of the 8 patients with distant lymph node metastases underwent radiotherapy, 2 patients underwent surgery, and 1 patient underwent both (radiotherapy and surgery) (Table 3).

No toxicity or complications related to the metastasisdirected therapy occurred. There was no difference between the two groups regarding the type of first-line endocrine treatment. The median metastasis-free interval was not different between the two groups ( 54.2 vs. 58.1 months, $p=0.773)$. There was a significant difference in metastatic site $(p=0.026)$, as the local treatment group had a higher rate of bone metastasis. The median number of metastases was not different between the two groups $(p=0.121)$, whereas the mean maximal tumor dimension was significantly greater in the local treatment group $(p=0.005)$.

\section{Survival Outcomes}

Progression-Free Survival. During the follow-up period, the disease progression rates after distant metastasis 
Table 3. Sites of metastases and local treatment according to localization

Type of local treatment for distant metastases ${ }^{\mathrm{a}}$

$\begin{array}{ll}\text { Sites of metastases } & \\ \text { Bone }(n=23) & \text { Radiotherapy }(n=22) \text {, surgery }(n=1) \\ \text { Lung }(n=2) & \text { Surgery }(n=2) \\ \text { Distant lymph node }(n=8) & \text { Radiotherapy }(n=5) \text {, surgery }(n=2) \text {, radiotherapy and surgery }(n=1)\end{array}$

a Surgery included resection of bone, wedge resection of lung, and lymph node dissection.

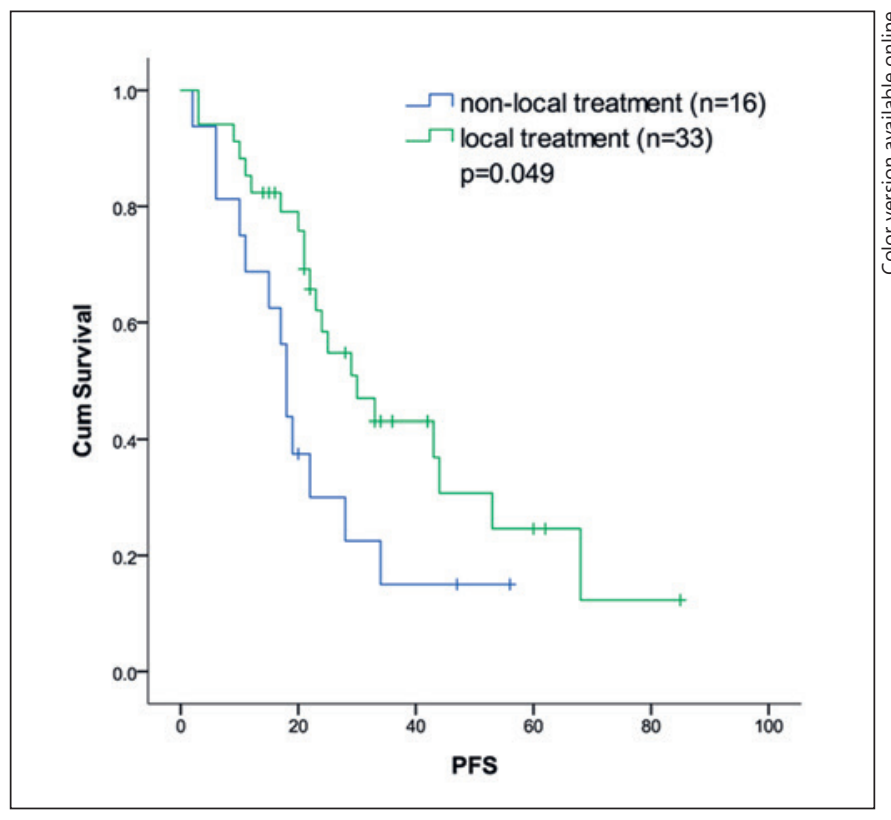

Fig. 1. Kaplan-Meier curve for progression-free survival (PFS) with regard to local treatment. Median PFS was significantly longer in the local treatment group than in the no local treatment group (30.0 vs. 18.0 months, $p=0.049$, log-rank test). Cum, cumulative.

were 60.6 and $81.3 \%$ in the local treatment group and the no local treatment group, respectively. The PFS rates were analyzed 1,2, and 3 years after metastasis. Median PFS was significantly longer in the local treatment group than in the no local treatment group (30.0 vs. 18.0 months, $p=$ 0.049 ; Fig. 1). In the local treatment group, the 1-, 2-, and 3 -year PFS rates were $81.3,59.1$, and $42.4 \%$, respectively. In the no local treatment group, the 1-, 2-, and 3-year PFS rates were $68.8,30.0$, and $15.0 \%$, respectively. Multivariate Cox regression analysis revealed local treatment to be a prognostic factor for longer PFS (hazard ratio [HR] 0.370, 95\% CI 0.164-0.834, $p=0.016$ ). In addition, metastasis to distant lymph nodes (HR 0.259, 95\% CI 0.088-0.765, $p=$ 0.014 ) and the number of pathologically positive axillary nodes (HR 1.077, 95\% CI 1.020-1.138, $p=0.008$ ) were independent factors affecting PFS (Table 4). In the subgroup analysis of patients with bone metastasis, the median PFS of the local treatment group and the no local treatment group was 24 and 18 months $(p=0.016$ ), respectively.

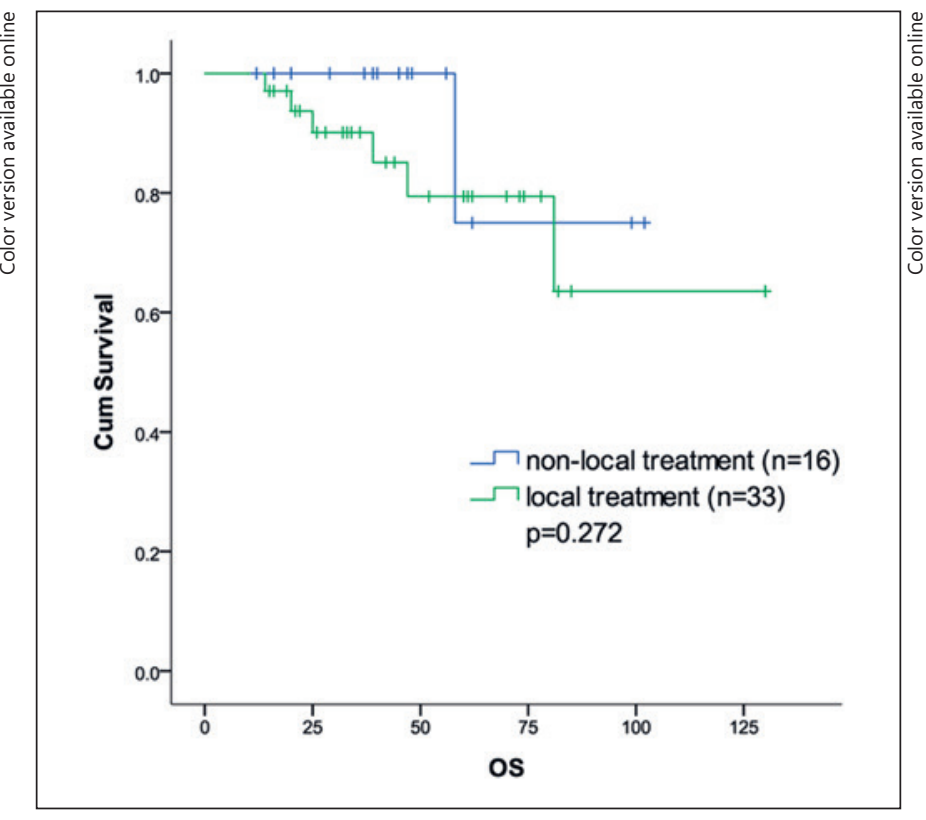

Fig. 2. Kaplan-Meier curve for overall survival (OS) after metastasis with regard to local treatment. The OS did not differ significantly with regard to local treatment ( $p=0.272$, log-rank test). Cum, cumulative.

OS after Metastasis. The mortality rates after distant metastasis were 18.2 and $6.3 \%$ in the local treatment and no local treatment groups, respectively. In the local treatment group, the 1-, 3-, and 5-year OS rates were 96.9, 89.4 , and $77.4 \%$, respectively. In the no local treatment group, the 1-, 3-, and 5-year OS rates were 100.0, 100.0, and $75.0 \%$, respectively. OS after metastasis was not different with regard to local treatment $(72.3 \mathrm{vs.} 91.0$ months, $p=0.272$; Fig. 2). The HR of local treatment was 4.186 (95\% CI 0.424-41.294, $p=0.220$ ).

\section{Discussion}

In the present study, we discovered potential benefits for PFS with local treatment of metastatic lesions in patients with recurrent HR-positive/HER2-negative oligometastatic breast cancer. Although OS did not differ with regard to metastasis-directed therapy, our findings sug- 
Table 4. Multivariate Cox regression analysis of progressionfree survival

\begin{tabular}{|c|c|c|}
\hline & \multicolumn{2}{|c|}{ Progression-free survival } \\
\hline & HR (95\% CI) & $p$ value \\
\hline Age & $1.026(0.986-1.067)$ & 0.201 \\
\hline Number of pathologically positive & & \\
\hline $\begin{array}{l}\text { axillary nodes } \\
\text { Initial stage } 3 \text { (ref: initial stage } 1 \text { ) }\end{array}$ & $\begin{array}{l}1.077(1.020-1.138) \\
1.451(0.175-12.038)\end{array}$ & 0.730 \\
\hline MFI & $0.993(0.983-1.002)$ & 0.144 \\
\hline $\begin{array}{l}\text { Distant lymph node metastasis } \\
\text { (reference: bone metastasis) }\end{array}$ & $0.259(0.088-0.765)$ & $0.014^{*}$ \\
\hline Number of metastases & $1.320(0.559-3.120)$ & 0.526 \\
\hline Local treatment & $0.370(0.164-0.834)$ & $0.016^{*}$ \\
\hline
\end{tabular}

MFI, metastasis-free interval; HR, hazard ratio; CI, confidence interval. ${ }^{*} p<0.05$.

gest that metastasis-directed therapy for metastatic lesions could prolong the duration of disease control without progression in oligometastatic patients. Despite the small sample size and selection bias of a retrospective study, our findings highlight the potential relevance of metastasis-directed therapy for a specific subset of oligometastatic patients in terms of PFS.

Previous studies have reported that local treatment may be beneficial to oligometastatic breast cancer patients. It is, however, clinically important to identify candidates who could benefit from local treatment among patients with oligometastasis. Although two previous studies suggested that patients with a disease-free interval greater than 36 months could be candidates for local treatment, data regarding which population of patients could benefit from local treatment in addition to systemic treatment are lacking $[25,26]$.

Here, we narrowed down the definition of oligometastasis and found a positive effect of local treatment on PFS for 1 or 2 metastatic lesions in a single organ with a maximal diameter of $\leq 3 \mathrm{~cm}$, suggesting that metastatic lesions meeting these criteria are suitable for surgical resection. Moreover, in patients with HR-positive/HER2-negative metastatic breast cancer, we found that current systemic therapy, which is mainly based on endocrine therapy and cyclin-dependent kinase 4/6 (CDK4/6) inhibitor, is very effective in halting disease progression [3] Hence, we postulated that local treatment has the potential to offer clinical benefits to HR-positive/HER2-negative patients considering our inclusion criteria.

Recent trials have sought to improve PFS through the addition of new drugs to endocrine therapy in HR-positive/HER2-negative metastatic breast cancer [27-29]. These innovative targeted drugs, including mTOR (mammalian target of rapamycin) inhibitor and CDK4/6 in- hibitor, have succeeded in improving PFS, compared to conventional endocrine therapy. In the PALOMA-3 trial, among patients with advanced HR-positive/HER2-negative breast cancer who exhibited sensitivity to endocrine therapy, those who received palbociclib in addition to fulvestrant had longer OS than those treated with fulvestrant alone [3]. However, there was no OS benefit with the addition of palbociclib for all patients of the PALOMA-3 trial. Moreover, in the BOLERO-2 trial, adding mTOR inhibitor did not elicit a significant improvement in OS, despite a statistically significant improvement in PFS (4.6-month prolongation of PFS) [27].

The reason why no significant OS benefit has been observed may be that uncontrolled post-study treatments, including chemotherapy, after disease progression might act as a potential confounding factor for final survival analyses. Since it is difficult to demonstrate an improved OS for new interventions in HR-positive/HER2-negative metastatic breast cancer, PFS could be a reasonable endpoint with which to evaluate the effects of metastasis-directed therapy for these patients. Indeed, OS did not differ with regard to local treatment in our patients; in our study, deaths were more frequent in the local treatment group than in the no local treatment group. Uncontrolled post-progression therapy might be one reason for a lack of benefit for OS in the local treatment group. In addition, as patients with a low volume of HR-positive/HER2-negative metastatic breast cancer could survive a relatively long time $[1,2]$, it would be more difficult to find an improvement in OS such as that seen in the PALOMA- 3 or BOLERO-2 trials. To observe an OS benefit in HR-positive/HER2-negative metastatic breast cancer patients, more large-sized prospective trials with a long-term follow-up would be required.

Currently, guidelines on the timing of metastasis-directed therapy initiation in patients with oligometastasis are not available. It remains controversial whether performing metastasis-directed therapy initially or later is to be guided by responses to initial systemic therapy. The study by Hanrahan et al. [30] reported a clinical benefit of metastasis-directed therapy as a first-line therapy after the diagnosis of oligometastasis. In our study, local treatment was performed at the initial diagnosis of oligometastasis.

According to the 4th International Consensus Guidelines for Advanced Breast Cancer (ABC4), 91\% of the experts agreed that local treatment could be considered at any time point in the course of the disease with the aim of achieving complete remission in oligometastatic patients [9]. Despite this consensus guideline, the benefit of metastasis-directed therapy is still controversial. Our retrospective findings showed a benefit of metastasis-directed therapy only in terms of delaying disease progression but did not provide evidence of improving OS. Meanwhile, however, a large randomized prospective trial is ongoing to ad- 
dress this controversial issue [31]. The NRG-BR002 study is a randomized clinical phase II trial evaluating whether stereotactic body radiotherapy and/or surgical resection of all metastatic sites will improve PFS in newly developed oligometastatic patients. If a positive signal regarding PFS can be identified, the trial will migrate to phase III in order to demonstrate an OS benefit with localized treatments for metastatic lesions. The eligibility criteria for the trial are enrollment within 1 year of metastatic diagnosis while undergoing first-line systemic therapy without progression.

No toxicity or complications related to local treatment occurred in the present study. Although data on toxicity or complications after local treatment in breast cancer patients with oligometastasis are still lacking, no toxicities of grade $\geq 3$ have previously been reported [32]. Future prospective studies on adverse effects of metastasis-directed therapy may be necessary for a risk-benefit analysis.

A major limitation of our study is the small sample size due to the strict inclusion criteria. Additionally, a selection bias may exist because of the nature of retrospective analyses. Actually, the group with local treatment had more metastatic disease in the bones and distant lymph nodes, whereas they had fewer hepatic metastases. Despite these limitations, we were able to address the clinical benefits of metastasis-directed therapy in this specific subpopulation of patients in terms of PFS. Moreover, our study has a strength, since the data were collected from multiple institutions, which could be more representative than data from a single institution.

\section{Conclusions}

Local treatment could positively affect disease progression in patients with HR-positive/HER2-negative oligometastatic breast cancer. A prospective trial is warranted to answer further questions about the role of metastasis-directed therapy in oligometastatic breast cancer.

\section{Statement of Ethics}

This study was approved by the Institutional Ethics Committee of Gangnam Severance Hospital. No consent was necessary due to the retrospective study design.

\section{Disclosure Statement}

The authors declare no conflicts of interest.

\section{Funding Sources}

There was no funding for this study.

\section{Author Contributions}

C.C., S.G.A., and J.J. designed the research; T.-K.Y., K.M.K., S.J.B., C.Y., S.P., and J.S. provided the data; C.C., S.G.A., and J.J. wrote the manuscript; J.J. had the final responsibility for the decision to submit the manuscript for publication.

\section{References}

1 Malmgren JA, Mayer M, Atwood MK, Kaplan HG. Differential presentation and survival of de novo and recurrent metastatic breast cancer over time: 1990-2010. Breast Cancer Res Treat. 2018 Jan;167(2):579-90.

2 Caswell-Jin JL, Plevritis SK, Tian L, Cadham CJ, Xu C, Stout NK, et al. Change in Survival in Metastatic Breast Cancer with Treatment Advances: Meta-Analysis and Systematic Review. JNCI Cancer Spectr. 2018 Nov; 2(4):pky062.

3 Turner NC, Slamon DJ, Ro J, Bondarenko I, Im SA, Masuda N, et al. Overall Survival with Palbociclib and Fulvestrant in Advanced Breast Cancer. N Engl J Med. 2018 Nov; 379(20):1926-36.

4 André F, Ciruelos E, Rubovszky G, Campone M, Loibl S, Rugo HS, et al.; SOLAR-1 Study Group. Alpelisib for PIK3CA-Mutated, Hormone Receptor-Positive Advanced Breast Cancer. N Engl J Med. 2019 May;380(20): 1929-40.

5 Swain SM, Kim SB, Cortés J, Ro J, Semiglazov $\mathrm{V}$, Campone M, et al. Pertuzumab, trastuzumab, and docetaxel for HER2-positive metastatic breast cancer (CLEOPATRA study): overall survival results from a randomised, double-blind, placebo-controlled, phase 3 study. Lancet Oncol. 2013 May;14(6):461-71.
6 Mahner S, Schirrmacher S, Brenner W, Jenicke L, Habermann CR, Avril N, et al. Comparison between positron emission tomography using 2-[fluorine-18]fluoro-2-deoxy-Dglucose, conventional imaging and computed tomography for staging of breast cancer. Ann Oncol. 2008 Jul;19(7):1249-54.

7 Trovo M, Furlan C, Polesel J, Fiorica F, Arcangeli S, Giaj-Levra N, et al. Radical radiation therapy for oligometastatic breast cancer: results of a prospective phase II trial. Radiother Oncol. 2018 Jan;126(1):177-80.

8 Hellman S, Weichselbaum RR. Oligometastases. J Clin Oncol. 1995 Jan;13(1):8-10.

9 Cardoso F, Senkus E, Costa A, Papadopoulos E, Aapro M, André F, et al. 4th ESO-ESMO International Consensus Guidelines for Advanced Breast Cancer (ABC 4). Ann Oncol. 2018 Aug;29(8):1634-57.

10 Buzdar AU, Blumenschein GR, Montague ED, Hortobagyi GN, Yap HY, Pinnamaneni $\mathrm{K}$, et al. Combined modality approach in breast cancer with isolated or multiple metastases. Am J Clin Oncol. 1984 Feb;7(1):45-50.

11 Planchard D, Soria JC, Michiels S, Grunenwald D, Validire P, Caliandro R, et al. Uncertain benefit from surgery in patients with lung metastases from breast carcinoma. Cancer. 2004 Jan;100(1):28-35.
12 Friedel G, Pastorino U, Ginsberg RJ, Goldstraw $\mathrm{P}$, Johnston $\mathrm{M}$, Pass $\mathrm{H}$, et al.; International Registry of Lung Metastases, London, England. Results of lung metastasectomy from breast cancer: prognostic criteria on the basis of 467 cases of the International Registry of Lung Metastases. Eur J Cardiothorac Surg. 2002 Sep;22(3):335-44.

13 Kirkinis MN, Lyne CJ, Wilson MD, Choong PF. Metastatic bone disease: a review of survival, prognostic factors and outcomes following surgical treatment of the appendicular skeleton. Eur J Surg Oncol. 2016 Dec;42(12): 1787-97.

14 Adam R, Aloia T, Krissat J, Bralet MP, Paule $\mathrm{B}$, Giacchetti S, et al. Is liver resection justified for patients with hepatic metastases from breast cancer? Ann Surg. 2006 Dec;244(6): 897-907.

15 Jandial R, Hoshide R, Waters JD, Somlo G. Operative and Therapeutic Advancements in Breast Cancer Metastases to the Brain. Clin Breast Cancer. 2018 Aug; 18(4):e455-67.

16 Hoffmann K, Franz C, Hinz U, Schirmacher P, Herfarth C, Eichbaum M, et al. Liver resection for multimodal treatment of breast cancer metastases: identification of prognostic factors. Ann Surg Oncol. 2010 Jun;17(6): 1546-54. 
17 Bathe OF, Kaklamanos IG, Moffat FL, Boggs J, Franceschi D, Livingstone AS. Metastasectomy as a cytoreductive strategy for treatment of isolated pulmonary and hepatic metastases from breast cancer. Surg Oncol. 1999 Jul;8(1):35-42.

18 Pagani O, Senkus E, Wood W, Colleoni M, Cufer T, Kyriakides S, et al.; ESO-MBC Task Force. International guidelines for management of metastatic breast cancer: can metastatic breast cancer be cured? J Natl Cancer Inst. 2010 Apr;102(7):456-63.

19 Salama JK, Hasselle MD, Chmura SJ, Malik R, Mehta N, Yenice KM, et al. Stereotactic body radiotherapy for multisite extracranial oligometastases: final report of a dose escalation trial in patients with 1 to 5 sites of metastatic disease. Cancer. 2012 Jun;118(11):2962-70.

20 Kang JK, Kim MS, Kim JH, Yoo SY, Cho CK, Yang KM, et al. Oligometastases confined one organ from colorectal cancer treated by SBRT. Clin Exp Metastasis. 2010 Apr;27(4):273-8.

21 Stinauer MA, Kavanagh BD, Schefter TE, Gonzalez R, Flaig T, Lewis K, et al. Stereotactic body radiation therapy for melanoma and renal cell carcinoma: impact of single fraction equivalent dose on local control. Radiat Oncol. 2011 Apr;6(1):34

22 Tree AC, Khoo VS, Eeles RA, Ahmed M, Dearnaley DP, Hawkins MA, et al. Stereotactic body radiotherapy for oligometastases. Lancet Oncol. 2013 Jan;14(1):e28-37.
23 National Comprehensive Cancer Network. NCCN clinical practice guidelines in oncology: breast cancer, version 2 [Internet]. 2018. Available from: https://www.nccn.org/professionals/physician_gls/pdf/breast.pdf.

24 Rugo HS, Rumble RB, Macrae E, Barton DL, Connolly HK, Dickler MN, et al. Endocrine therapy for hormone receptor-positive metastatic breast cancer: American Society of Clinical Oncology Guideline. J Clin Oncol. 2016 Sep;34(25):3069-103.

25 Abbott DE, Brouquet A, Mittendorf EA, Andreou A, Meric-Bernstam F, Valero V, et al. Resection of liver metastases from breast cancer: estrogen receptor status and response to chemotherapy before metastasectomy define outcome. Surgery. 2012 May;151(5):710-6.

26 Sledge GW Jr. Curing Metastatic Breast Cancer. J Oncol Pract. 2016 Jan;12(1):6-10.

27 Piccart M, Hortobagyi GN, Campone M, Pritchard KI, Lebrun F, Ito Y, et al. Everolimus plus exemestane for hormone-receptorpositive, human epidermal growth factor receptor-2-negative advanced breast cancer: overall survival results from BOLERO-2. Ann Oncol. 2014 Dec;25(12):2357-62.

28 Finn RS, Martin M, Rugo HS, Jones S, Im SA, Gelmon K, et al. Palbociclib and Letrozole in Advanced Breast Cancer. N Engl J Med. 2016 Nov;375(20):1925-36.
29 Hortobagyi GN, Stemmer SM, Burris HA, Yap YS, Sonke GS, Paluch-Shimon S, et al. Updated results from MONALEESA-2, a phase III trial of first-line ribociclib plus letrozole versus placebo plus letrozole in hormone receptor-positive, HER2-negative advanced breast cancer. Ann Oncol. 2018 Jul;29(7): 1541-7.

30 Hanrahan EO, Broglio KR, Buzdar AU, Theriault $\mathrm{RL}$, Valero $\mathrm{V}$, Cristofanilli $\mathrm{M}$, et al. Combined-modality treatment for isolated recurrences of breast carcinoma: update on 30 years of experience at the University of Texas M.D. Anderson Cancer Center and assessment of prognostic factors. Cancer. 2005 Sep; 104(6):1158-71.

31 Chmura SJ, Winter KA, Salama JK, Woodward WW, Borges V, Al-Hallaq $\mathrm{H}$, et al. NRG-BR002: a phase IIR/III trial of standard of care therapy with or without stereotactic body radiotherapy (SBRT) \&/or surgical ablation for newly oligometastatic breast cancer. Cancer Res. 2017;77(4 Suppl):abstract OT104-06.

32 Corbin KS, Ranck MC, Hasselle MD, Golden DW, Partouche J, Wu T, et al. Feasibility and toxicity of hypofractionated image guided radiation therapy for large volume limited metastatic disease. Pract Radiat Oncol. 2013 OctDec;3(4):316-22. 